\title{
PRONORMALITY, CONTRANORMALITY AND GENERALIZED NILPOTENCY IN INFINITE GROUPS
}

\author{
Leonid A. Kurdachenko and Igor Ya. Subbotin
}

$\begin{array}{ll}\text { Abstract } & \text { This article is dedicated to some criteria of generalized nilpo- } \\ & \text { tency involving pronormality and abnormality. Also new results } \\ & \text { on groups, in which abnormality is a transitive relation, have been } \\ & \text { obtained. }\end{array}$

\section{Introduction}

There is a variety of very practical characterizations of nilpotency in finite groups. However, we are losing a majority of them at the transition to infinite groups. In this case, these characterizations are forming the bases for distinct definitions of new classes of infinite generalized nilpotent groups. On the other hand, for every concrete characterization of finite nilpotent groups it is very interesting to determine the limits in which these characterizations are still valid; or more generally to define the boundaries in which a certain characterization leads to a certain class of generalized nilpotent groups. One of the most suitable classes on which many different characterizations of nilpotency have been extended is the class of finitely generated soluble groups. The main reason for this is the famous Robinson's Theorem (see, for example, [RD 2, Theorem 10.51]) proving that if every finite factor-group of a finitely generated soluble group $G$ is nilpotent, then $G$ is nilpotent. Among other "good" classes we can mention the class of Chernikov groups and the class of minimax groups. These classes are not far from finite groups because they satisfy very strong finiteness conditions. Nevertheless, there are some classes of infinite groups, which are far enough from finite groups and satisfy some distinct criteria of generalized nilpotency such that locally nilpotency, hypercentrality, the normalizer condition, and so on. The class of (locally nilpotent)-by-finite groups and the class

2000 Mathematics Subject Classification. 20F19, 20F24, $20 \mathrm{E} 34$.

Key words. FC-hypercentral groups, FC-nilpotent groups, nilpotency criteria, contranormal subgroups, pronormal subgroups, abnormal subgroups, transitive relations. 
of $F C$-groups serve as such examples. Recall, that a group $G$ is called an FC-group, if the conjugate class $x^{G}=\left\{x^{g} \mid g \in G\right\}$ is finite for each element $x \in G$. The class of $F C$-groups is unique in some certain sense: many fundamental properties (particularly concerning with some criteria of nilpotency) of finite groups have been naturally extended to $F C$-groups. For example, we can mention the following results.

If every subgroup of an FC-group $G$ is subnormal, then $G$ is nilpotent (see, for example [SH 2]).

If an FC-group $G$ is a direct product of its Sylow p-subgroups for all prime $p$, then $G$ is hypercentral.

If an FC-group $G$ satisfies the normalizer condition, then $G$ is hypercentral.

If every maximal subgroup of an $F C$-group $G$ is normal, then $G$ is hypercentral.

If $G$ is an FC-group and $[G, G] \leq \operatorname{Fratt}(G)$, then $G$ is hypercentral.

Last three statements are consequences of the following well-known fact.

If for an FC-group $G$ the factor-group $G / \zeta(G)$ is residually nilpotent, then $G$ is hypercentral.

Therefore it is natural to find such generalizations of the class of $F C$-groups, on which these and other conditions of hypercentrality could be extended.

Let $G$ be a group. Put $F C(G)=\left\{x \in G \mid x^{G}\right.$ is finite $\}$. It is easy to see that $F C(G)$ is a characteristic subgroup of $G$. The subgroup $F C(G)$ is called the $F C$-center of $G$. A group $G$ is an FC-group if and only if $G=F C(G)$.

Starting from the $F C$-center we can construct the upper FC-central series of a group $G$ :

$$
\langle 1\rangle=C_{0} \leq C_{1} \leq \cdots \leq C_{\alpha} \leq C_{\alpha+1} \leq \cdots \leq C_{\gamma}
$$

where $C_{1}=F C(G), C_{\alpha+1} / C_{\alpha}=F C\left(G / C_{\alpha}\right), \alpha<\gamma$, and $F C\left(G / C_{\gamma}\right)=\langle 1\rangle$.

The last term $C_{\gamma}$ of this series is called the upper FC-hypercenter. If $C_{\gamma}=G$, then the group $G$ is called FC-hypercentral; if $\gamma$ is finite, then $G$ is called FC-nilpotent. 
Consider extensions of the criteria listed above on $F C$-nilpotent or $F C$-hypercentral groups.

Let $G$ be a group, every subgroup of which is subnormal. Then $G$ is locally nilpotent (see, for example, [LS, Theorem 2.5.1]). Therefore if $G$ is $F C$-hypercentral, then $G$ is hypercentral. Note that there exists an $F C$-nilpotent non-nilpotent group $G$, every subgroup of which is subnormal [SH 1]. For some subclasses of $F C$-hypercentral groups the situation looks much better: if, for example, $G$ is a periodic $F C$-hypercentral group with all subgroup subnormal, then $G$ is nilpotent (it follows from the main result of the paper [MW]); if $G$ is a torsion-free group with all subgroup subnormal, then $G$ is nilpotent [SH 3].

Let $G$ be a group satisfying the normalizer condition. Then $G$ is locally nilpotent $[\mathbf{P B}]$. Therefore if $G$ is $F C$-hypercentral, then $G$ is hypercentral. Groups with the normalizer condition can be characterized with the help of the concept of the ascending subgroup. Recall, that a subgroup $H$ of a group $G$ is called ascending if there is an ascending series

$$
H=H_{0} \leq H_{1} \leq \cdots \leq H_{\alpha} \leq H_{\alpha+1} \leq \cdots \leq H_{\gamma}=G
$$

such that $H_{\alpha}$ is a normal subgroup of $H_{\alpha+1}$ for all $\alpha<\gamma$. It is wellknown that a group $G$ satisfies the normalizer condition if and only if every subgroup of $G$ is ascending [BR, Theorem 4.13]. The dual to the idea of an ascending subgroup is the concept of a descending subgroup. Let $H$ be a subgroup of a group $G$. We construct the normal closure series in the following way.

Put $H_{1}=H^{G}$. If for an ordinal $\alpha$ the subgroups $H_{\beta}$ have been already constructed for all $\beta<\alpha$, then put $H_{a}=\cap_{\beta<\alpha} H_{\beta}$ whenever $\alpha$ is a limit ordinal. If $\alpha-1$ exists and $K=H_{\alpha-1}$, then put $H_{\alpha}=H^{K}$. There is an ordinal $\gamma$ such that $H_{\gamma}=H_{\gamma+1}$. In other words, $H^{H_{\gamma}}=H \gamma$. A subgroup $H$ is called descending, if the last term of the normal closure series coincides with $H$.

Both ascending and descending subgroups are generalizations of subnormal subgroups. With the help of these concepts we obtain the following conditions of hypercentrality for a group.

Theorem A. Let $G$ be a group every subgroup of which is descending. If $G$ is $F C$-hypercentral, then $G$ is hypercentral.

A subgroup $H$ is contranormal in a group $G$ if $H^{G}=G[\mathbf{R} \mathbf{1}]$. The following criterion of nilpotency of finite groups involves contranormality. 
A finite group is nilpotent if and only if it has no proper contranormal subgroups.

Further we will also consider criteria of (locally) nilpotency involving pronormal and abnormal subgroups. These subgroups introduced by P. Hall and R. W. Carter respectively in the study of nilpotency in finite groups. Pronormal subgroups play the main role in generalizations both normality and Sylow properties (see for example, the survey $[\mathbf{B B}]$ and papers $[\mathbf{F}],[\mathbf{C}],[\mathbf{P}],[\mathbf{M}],[\mathbf{R} \mathbf{2}],[\mathbf{W}],[\mathbf{K S} \mathbf{1}],[\mathbf{K S} \mathbf{2}],[\mathbf{K S} \mathbf{3}],[\mathbf{S K}]$, $[\mathbf{d e F K S}],[\mathbf{S E}])$. Recall that a subgroup $H$ of a group $G$ is pronormal in $G$, if $H$ and $H^{g}$ are conjugate in $\left\langle H, H^{g}\right\rangle$ for each element $g \in G$. A subgroup $H$ of a group $G$ is abnormal in $G$, if $g \in\left\langle H, H^{g}\right\rangle$ for each element $g \in G$.

We will also obtain some generalizations of the following criteria of nilpotency of finite groups:

A finite group is nilpotent if and only if all its pronormal subgroups are normal.

Note that in the class of locally nilpotent groups all pronormal subgroups are normal [KS 3]. It is easy to see that the condition: "every pronormal subgroup of a group $G$ is normal" is equivalent to the condition " $G$ has no proper abnormal subgroups". It follows from the fact that a normalizer of a pronormal subgroup is an abnormal subgroup (see some elementary properties of pronormality and abnormality listed at the beginning of Section 3). So we come to the following criterion:

A finite group is nilpotent if and only if it has no proper abnormal subgroups.

As many others these criteria could be extended on $F C$-groups (see Proposition 3.4).

The following theorem is one of the main our results.

Theorem B. Let $G$ be a group, $A$ a normal abelian subgroup of $G$ such that $A \leq F C(G)$ and $G / A$ is an $F C$-group.

(1) If every pronormal subgroup of $G$ is normal, then $G$ is hypercentral.

(2) If $G$ does not include proper contranormal subgroups, then $G$ is hypercentral.

(3) If $G$ does not include proper abnormal subgroups, then $G$ is hypercentral.

For periodic groups these results could be straightened significantly. 
Theorem C. Let $G$ be a periodic group, the FC-center of which includes a $G$-invariant subgroup $H$ such that $G / H$ is a hypercentral group.

(1) If every pronormal subgroup of $G$ is normal, then $G$ is hypercentral.

(2) If $G$ does not include proper contranormal subgroups, then $G$ is hypercentral.

(3) If $G$ does not include proper abnormal subgroups, then $G$ is hypercentral.

Corollary C1. Let $G$ be an FC-nilpotent periodic group.

(1) If every pronormal subgroup of $G$ is normal, then $G$ is hypercentral.

(2) If $G$ does not include proper contranormal subgroups, then $G$ is hypercentral.

(3) If $G$ does not include proper abnormal subgroups, then $G$ is hypercentral.

A. Ballester-Bolinches and T. Pedraza $[\mathbf{B}-\mathbf{B P}]$ have considered radical periodic groups with Chernikov Sylow $p$-subgroups for all primes $p$, which have no proper contranormal subgroups. The class of these groups they denoted by $\mathcal{B}$. Note that some results (in particular, Theorem 6 ) of this paper can be extended on the class of periodic locally soluble groups with Chernikov Sylow $p$-subgroups for all prime $p$ (see Corollaries C2, C3).

It is interesting to admit that contranormality possesses the property of transitivity. However, simple examples show that normality, pronormality, and abnormality in general are not transitive (see, for example, $[\mathbf{B B}])$. The class of groups in which normality is a transitive relation ( $T$-groups) is well studied now. These groups and some their generalizations has been investigated by many authors. The most valuable results about $T$-groups one can find in [RD 1]. The class of soluble groups with transitivity of pronormality is fully described in the recent paper [KUS]. As it follows from Theorem D below, the class of soluble groups in which abnormality is transitive ( $S T A$-groups) is much wider and contains both classes of soluble groups with transitive normality and transitive pronormality ( $S T$ - and $S T P$-groups respectively). More specifically, based on Theorem D and using [RD 1, Theorem 2.3.1] and [KUS, Theorem 2] one can easily check the following strict embeddings

$$
\{S T P \text {-groups }\} \subset\{S T \text {-groups }\} \subset\{S T A \text {-groups }\} \text {. }
$$


Theorem D. Let a group $G$ includes a Dedekind normal subgroup $H$ such that $G / H$ has no proper abnormal subgroup. Then the abnormality is a transitive relation in $G$.

Corollary D1. Let a group $G$ includes an abelian normal subgroup $H$ such that $G / H$ has no proper abnormal subgroups. Then the abnormality is a transitive relation in $G$.

Corollary D2. Let a group $G$ includes an abelian normal subgroup $H$ such that $G / H$ is hypercentral. Then the abnormality is a transitive relation in $G$.

Corollary D3. Every metabelian group is a group in which abnormality is a transitive relation.

Corollary D4. In soluble groups in which normality is a transitive relation abnormality also is a transitive relation.

\section{On direct decompositions of $\mathrm{ZG}$-modules connected to some normal subgroups}

Let $G$ be a hypercentral group, $H$ a normal subgroup of $G, A$ a $\mathbf{Z} G$-module. Put $\zeta_{\mathbf{Z} H}(A)=\{a \in A \mid$ ax $=$ afor each $x \in H\}$. It is easy to see that $\zeta_{\mathbf{Z} H}(A)$ is a $\mathbf{Z} G$-submodule of $A$. $\zeta_{\mathcal{Z} H}(A)$ is called the $\mathbf{Z} H$-center of $A$. Starting from the $\mathbf{Z} H$-center, we can construct the upper $\mathbf{Z} H$-central series of $A$

$$
\langle 0\rangle \leq C_{0} \leq C_{1} \leq \cdots \leq C_{\alpha} \leq C_{\alpha+1} \leq \cdots \leq C_{\gamma}
$$

by the following rule: $C_{1}=\zeta_{\mathbf{z}_{H}}(A), C_{\alpha+1} / C_{\alpha}=\zeta_{Z_{H}}\left(A / C_{\alpha}\right), \alpha<\gamma$, $\zeta_{\mathcal{Z} H}\left(A / C_{\gamma}\right)=\langle 0\rangle$. The last term $C_{\gamma}$ of this series is called the upper $\mathbf{Z} H$-hypercenter of $A$.

Let $B, C$ be some $\mathbf{Z} G$-submodules of $A, B \leq C$. The factor $C / B$ is called $H$-central (respectively $H$-eccentric) if $C_{H}(C / B)=H$ (respectively $\left.C_{H}(C / B) \neq H\right)$.

Lemma 2.1. Let $G$ be a hypercentral group, $H$ a non-identity normal subgroup of $G, A$ a $\mathcal{Z} G$-module, $C_{G}(A)=\langle 1\rangle, B$ a $\mathbf{Z} G$-submodule satisfying the following conditions:

(1) $B$ and $A / B$ are simple $\mathbf{Z} G$-modules.

(2) $B$ is $H$-central, $A / B$ is $H$-eccentric.

Then there is a $\mathbf{Z} G$-submodule $C$ such that $A=B \oplus C$.

Proof: Since $G$ is a hypercentral group, $H \cap \zeta(G) \neq\langle 1\rangle$. Let $1 \neq z \in$ $H \cap \zeta(G)$. Consider the mapping $\phi_{z}: a \rightarrow a(z-1), a \in A$. The mapping $\phi_{z}$ is a $\mathcal{Z} G$-endomorphism by the selection of $z$, in particular, $\operatorname{Im} \phi_{z}$ 
and $\operatorname{Ker} \phi_{z}$ are $\mathcal{Z} G$-submodules of $A$. Moreover, $\operatorname{Ker} \phi_{z} \geq B$. If we assume that $\operatorname{Ker} \phi_{z} \neq B$, then $\operatorname{Ker} \phi_{z}=A$, that is $z \in C_{G}(A)=\langle 1\rangle$. Contradiction. Hence $\operatorname{Ker} \phi_{z}=B$, that is $A / \operatorname{Ker} \phi_{z} \cong \operatorname{Im} \phi_{z}=C$ is a $\mathcal{Z} G$-submodule of $A$ such that $C_{H}\left(\operatorname{Im} \phi_{z}\right) \neq H$. Moreover, $C$ is simple $\mathcal{Z} G$-submodule, therefore $C \cap B=\langle 0\rangle$, because $C \neq B$. Since $A / B$ is a simple $\mathcal{Z} G$-module, $C+B=A$, that is $A=B \oplus C$.

Lemma 2.2. Let $G$ be a hypercentral group, $H$ a non-identity normal subgroup of $G, H$ a $\mathcal{Z} G$-module, $C_{G}(A)=\langle 1\rangle, B$ a $\mathcal{Z} G$-submodule satisfying the following conditions:

(1) $B$ and $A / B$ are simple $\mathcal{Z} G$-modules.

(2) $A / B$ is $H$-central, $B$ is $H$-eccentric.

Then there is a $\mathcal{Z} G$-submodule $C$ such that $A=B \oplus C$.

Proof: Since $G$ is a hypercentral group, $H \cap \zeta(G) \neq\langle 1\rangle$. Let $1 \neq z \in$ $H \cap \zeta(G)$. The mapping $\phi_{z}: a \rightarrow a(z-1), a \in A$, is a $\mathcal{Z} G$-endomorphism of $A$. In particular, $\operatorname{Im} \phi_{z}$ and $\operatorname{Ker} \phi_{z}$ are $\mathcal{Z} G$-submodules of $A$. Since $C_{H}(A / B)=H, \operatorname{Im} \phi_{z} \leq B$. If we assume that $\operatorname{Im} \phi_{z}=\langle 0\rangle$, then $z \in C_{G}(A)=\langle 1\rangle$. Contradiction. Hence $\operatorname{Im} \phi_{z} \neq\langle 0\rangle$. Since $B$ is a simple $\mathcal{Z} G$-submodule, $\operatorname{Im} \phi_{z}=B$. The $\mathcal{Z} G$-factor $A(z-1) / B(z-1)$ is a $Z G$-homomorphic image of $A / B$. Since $C_{H}(A / B)=H, C_{H}(A(z-$ $1) / B(z-1))=H$. Since $B$ is a simple $\mathcal{Z} G$-submodule, from $A(z-1)=$ $\operatorname{Im} \phi_{z}=B$ and $C_{H}(B) \neq H$ we obtain the equality $A(z-1)=B(z-1)$. Hence for every $a \in A$ we have $a(z-1)=b(z-1)$ for some $b \in B$. It follows that $(a-b)(z-1)=0$, that is $a-b \in C_{A}(z)$ or $A=B+C_{A}(z)$. Since $C_{A}(z)=\operatorname{Ker} \phi_{z}, C_{A}(z)$ is a $\mathcal{Z} G$-submodule. Since $B$ is a simple $\mathcal{Z} G$-submodule, then either $B \cap C_{A}(z)=\langle 0\rangle$ or $B \leq C_{A}(z)$. In the last case $A=C_{A}(z)$, that is $z \in C_{G}(A)=\langle 1\rangle$, which is impossible. It follows that $B \cap C_{A}(z)=\langle 0\rangle$ and $A=B \oplus C$ where $C=C_{A}(z)$.

Let $G$ be a hypercentral group, $H$ a normal non-identity subgroup of $G, A$ a $\mathcal{Z} G$-module. $A \mathcal{Z} G$-submodule $C$ is called $H$-hypereccentric, if $C$ has an ascending series

$$
\langle 0\rangle \leq C_{0} \leq C_{1} \leq \cdots \leq C_{\alpha} \leq C_{\alpha+1} \leq \cdots \leq C_{\gamma}
$$

of $\mathcal{Z} G$-submodules of $A$ satisfying the following conditions:

$C_{\alpha+1} / C_{\alpha}$ is a simple $\mathcal{Z} G$-module.

$C_{\alpha+1} / C_{\alpha}$ is an $H$-eccentric factor for every $\alpha<\gamma$. 
Corollary 2.3. Let $G$ be a hypercentral group, $H$ a non-identity normal subgroup of $G, A$ a $\mathcal{Z} G$-module, having a finite $Z G$-composition series, $C_{G}(A)=\langle 1\rangle, C$ an upper $\mathcal{Z} H$-hypercenter of $A$. Then:

(1) $A=C \oplus E$ where $E$ is a maximal $H$-hypereccentric $\mathcal{Z} G$-submodule of $A$.

(2) $E$ includes every $H$-hypereccentric $\mathcal{Z} G$-submodule of $A$, in particular, this decomposition is unique.

Proof: Assertion (1) follows from Lemmas 2.1 and 2.2. Let $B$ be an $H$-hypereccentric $\mathcal{Z} G$-submodule of $A$. If $(B+E) / E$ is non-zero, it includes a non-zero simple $\mathcal{Z} G$-submodule $D / E$. Since $(B+E) / E \cong$ $B /(B \cap E), D / E$ is $\mathcal{Z} G$-isomorphic with some simple $\mathcal{Z} G$-factor of $B$. It follows that $C_{H}(D / E)=H$. On the other hand, $(B+E) / E \leq A / E \cong C$, that is $C_{H}(D / E)=H$. This contradiction proves that $B \leq E$.

Proposition 2.4. Let $G$ be a group, $A$ a normal abelian periodic subgroup of $G$ such that $A \leq F C(G)$ and $Q=G / C_{G}(A)$ is hypercentral. Let $H$ be a non-identity normal subgroup of $Q, C$ an upper $\mathcal{Z} H$-hypercenter of $A$. Then:

(1) $A=C \oplus E$ where $E$ is a maximal $H$-hypereccentric $\mathcal{Z} Q$-submodule of $A$.

(2) E includes every $H$-hypereccentric $\mathcal{Z} Q$-submodule of $A$; in particular, this decomposition is unique.

Proof: Since $A \leq F C(G)$ and $A$ is periodic, $A$ has a local family of finite $G$-invariant subgroups $\left\{A_{\lambda} \mid \lambda \in \Lambda\right\}$. Since $A_{\lambda}$ is finite, the $\mathcal{Z} Q$-submodule $A_{\lambda}$ has a finite composition $\mathcal{Z} Q$-series. By Corollary 2.3 $A_{\lambda}=C_{\lambda} \times E_{\lambda}$ where $C_{\lambda}$ is the upper $\mathcal{Z} H$-hypercenter of $A_{\lambda}$ (that is $\left.C_{\lambda}=C \cap A_{\lambda}\right), E_{\lambda}$ is a maximal $H$-hypereccentric $\mathcal{Z} Q$-submodule of $A_{\lambda}$, $\lambda \in \Lambda$. If $A_{\lambda} \leq A_{\mu}$, then by the isomorphism $A_{\lambda} /\left(A_{\lambda} \cap E_{\mu}\right) \cong_{\mathcal{Z} Q}$ $A_{\lambda} E_{\mu} / E_{\mu} \leq A m / E m \cong \mathcal{Z}_{Q} C_{\mu}$ we obtain that $A_{\lambda} \cap E_{\mu}$ is a maximal $H$-hypereccentric $\mathcal{Z} Q$-submodule of $A_{\lambda}$, that is $A_{\lambda} \cap E_{\mu}=E_{\lambda}$. Put $E=\cup_{\lambda \in \Lambda} E_{\lambda}$. Then $E$ is a $G$-invariant subgroup of $A$ such that $E \cap A_{\lambda}=E_{\lambda}, \lambda \in \Lambda$. By construction $E$ is an $H$-hypereccentric $\mathcal{Z} Q$-submodule of $A$. Since $A_{\lambda}=C_{\lambda} \times E_{\lambda}=\left(C \cap A_{\lambda}\right) \times\left(E \cap A_{\lambda}\right), A=C \times E$. In particular, $E$ is a maximal $H$-hypereccentric $\mathcal{Z} Q$-submodule of $A$. The proof of assertion (2) is the same as the relevant part of the proof of the previous corollary. 


\section{Proofs of main results. The non-periodic case}

For the reader convenience we are listing the following elementary properties of abnormal and pronormal subgroups (see, for example, $[\mathbf{B B}]$, $[\mathbf{D H}])$. We are going to use some of them in this paper.

Let $G$ be a group, $U$ a subgroup of $G$.

I. The following statements hold.

(1) If $A$ is pronormal in $G$ and $A \leq U$, then $A$ is pronormal in $U$.

(2) If $H$ is a normal subgroup of $G$, and $H \leq A$, then $A / H$ is pronormal in $G / H$ if an only if $A$ is pronormal in $G$.

(3) If $H$ is a normal subgroup of $G$ and $A$ is pronormal in $G$, then $A H / H$ is pronormal in $G / H$.

(4) If $H$ is a normal subgroup of $G$ and $A$ is pronormal in $G$, then $N_{G}(A H)=N_{G}(A) H$.

(5) If $A$ is pronormal in $G$ and $A$ is subnormal in $G$, then $A$ is normal in $G$.

(6) If $A$ is pronormal in $G$, then $N_{G}(A)$ is abnormal in $G$.

(7) If $A$ is pronormal in $G$, and $B$ is normal in $G$, then $A B$ is pronormal in $G$.

(8) If $G$ is a soluble group, then $A$ is pronormal in $G$ if and only if it satisfies the Frattini property. In particular, if $A$ is pronormal in $G$ and $H$ is a normal subgroup including $A$, then $G=N_{G}(A) H$.

(9) If $A$ is abnormal in $G$, then $A$ is abnormal in any intermediate subgroup of $G$.

II. The following statements are equivalent.

(1) $H$ is abnormal in $G$.

(2) $H$ is pronormal in $G$ and $N_{G}(H)=H$.

(3) $H$ is pronormal in $G$ and $N_{G}(K)=K$ for any intermediate subgroup $K$ for $H$.

III. Let $A$ be a subgroup of a group $G$ and $H$ be a $G$-invariant subgroup of $A$; then $A$ is abnormal in $G$ if and only if $A / H$ is abnormal in $G / H$.

The following Lemma 3.1 is well-known (see, for example, $[\mathbf{M c L}]$ ). 
Lemma 3.1. Let $G$ be an FC-hypercentral group. If $G$ is finitely generated, then $G$ is nilpotent-by-finite.

The following statements are almost obvious.

Lemma 3.2. Let $G$ be a group, every subgroup of which is descending.

(1) If $H$ is a subgroup of $G$, then every subgroup of $H$ is descending.

(2) If $L$ is a normal subgroup of $G$, then every subgroup of $G / L$ is descending.

(3) If $U / V$ is a section of $G$, then every subgroup of $U / V$ is descending.

Lemma 3.3. Let $G$ be a group, every subgroup of which is descending. Then every locally (soluble-by-finite) subgroup $H$ of $G$ is locally nilpotent.

Proof: Let $K$ is an arbitrary finitely generated subgroup of $H$ and let $S$ be a normal subgroup of finite index in $K$. By Lemma $3.2 \mathrm{~K} / \mathrm{S}$ is a finite group, every subgroup of which is descending. In other words, every subgroup of $K / S$ is subnormal. It follows that $K / S$ is nilpotent. Hence every finite factor-group of $K$ is nilpotent, so that $K$ is nilpotent [RD 2, Theorem 10.51].

Proof of Theorem A: By Lemma 3.1 the group $G$ is locally (nilpotentby-finite). Therefore we may use Lemma 3.3 and obtain that $G$ is locally nilpotent. Since $G$ is $F C$-hypercentral, $G$ is hypercentral.

Proposition 3.4. Let $G$ be an FC-group.

(1) If every pronormal subgroup of $G$ is normal, then $G$ is hypercentral.

(2) If $G$ does not include proper contranormal subgroups, then $G$ is hypercentral.

(3) If $G$ does not include proper abnormal subgroups, then $G$ is hypercentral.

Proof: The factor-group $G / \zeta(G)$ is residually finite and periodic (see, for example, [RD 2, Lemma 4.31]). Since a finite group, in which all pronormal subgroups are normal (respectively, a finite group, which does not include the proper contranormal subgroups) is nilpotent, $G / \zeta(G)$ is residually nilpotent. Since this factor-group is also periodic, $G / \zeta(G)$ is locally nilpotent. But a locally nilpotent $F C$-group is hypercentral (see, for example, [RD 2, Theorem 4.38]), so $G / \zeta(G)$, and therefore $G$, is hypercentral. 
Recall that a $\mathcal{Z} G$-module $A$ is called to be monolithic if the intersection of all non-zero $\mathcal{Z} G$-submodules of $A$ (the $\mathcal{Z} G$-monolith of $A$ ) is non-zero.

Lemma 3.5. Let a group $G$ includes a normal abelian periodic subgroup A satisfying the following conditions:

(1) $A \leq F C(G)$.

(2) $A$ is a monolithic $\mathcal{Z} G$-module with the $\mathcal{Z} G$-monolith $M$.

(3) $G / A$ is a hypercentral group.

(4) $G \neq C_{G}(M)$.

Then $A$ has an ascending series

$$
\langle 1\rangle=A_{0} \leq A_{1}=M \leq A_{2} \leq \cdots \leq A_{\alpha} \leq A_{\alpha+1} \leq \cdots \leq A_{\gamma}=A
$$

of $G$-invariant subgroups, every factor $A_{\alpha+1} / A_{\alpha}$ of which is $G$-chief and isomorphic to $M$ as a $\mathcal{Z} G$-module for each $\alpha<\gamma$.

Proof: Put $H=C_{G}(M)$. Since $M$ is a simple $\mathcal{Z} G$-submodule, condition (1) gives that $M$ is finite, so that $H$ is a normal subgroup of finite index. Since $G / H$ is nilpotent, it is a $p^{\prime}$-group (see, for example, [DH, Corollary B.9.4]). By Proposition 2.4 $A=C \times E$ where $C$ is an upper $\mathcal{Z} H$-hypercenter of $A$ and $E$ is a maximal $H$-hypereccentric $\mathcal{Z} G$-submodule of $A$. By the selection of $H$ we have $M \leq C$. Since $M$ is a $\mathcal{Z} G$-monolith of $A$, the equation $M \cap E=\langle 1\rangle$ implies that $E=\langle 1\rangle$. In other words, $A$ is $\mathcal{Z} H$-hypercentral.

Let $S / M=\operatorname{Soc}_{G}(A / M)$ be a subgroup, generated by all minimal $G$-invariant subgroups of $A / M$. Choose an arbitrary minimal $G$-invariant subgroup $U / M$ of $A / M$. Condition (1) yields that $U$ is finite, that is $V=$ $C_{G}(U)$ is a normal subgroup of finite index. If we assume that $V=H$, then by Maschke's Theorem (see, for example, [DH, Theorem A.11.4]) $U=M \times W$ for some $G$-invariant subgroup $W$. Since $A$ is monolithic, this is impossible. This contradiction proves that $V \neq H$. In particular, $H / V$ is a non-identity normal subgroup of the nilpotent group $G / V$, therefore $H / V \cap \zeta(G / V) \neq\langle 1\rangle$. Let $V \neq z V \in \zeta(G / V) \cap H / V$. Then the mapping $\phi: u \rightarrow[g, u], u \in U$, is a $\mathcal{Z} G$-endomorphism of $U$. Since $z \in H, M \leq \operatorname{Ker} \phi, \operatorname{Im} \phi=[g, U] \leq M$. Since $\operatorname{Ker} \phi \neq\langle 1\rangle, \operatorname{Ker} \phi=M$, $\operatorname{Im} \phi=M$, so that $U / \operatorname{Ker} \phi=U / M \cong_{\mathcal{Z} G} M$. Hence every minimal $G$-invariant subgroup of $S / M$ is isomorphic with $M$ as a $\mathcal{Z} G$-module. Since $A \leq F C(G), A$ has an upper $G$-socular series. Using the same arguments and transfinite induction, we prove this lemma. 
Lemma 3.6. Let a group $G$ includes a normal abelian periodic subgroup A satisfying the following conditions:

(1) $A \leq F C(G)$.

(2) $A$ is a monolithic $\mathcal{Z} G$-module with the $\mathcal{Z} G$-monolith $M$.

(3) $G / A$ is a hypercentral FC-group.

(4) $G \neq C_{G}(M)$.

Then $G$ includes a subgroup $S$ such that $G=A \lambda S$. Furthermore, every other complement to $A$ in $G$ is conjugate with $S$.

Proof: Put $H=C_{G}(M)$. Since $M$ is a simple $\mathcal{Z} G$-submodule, condition (1) implies that $M$ is finite, so that $H$ is a normal subgroup of finite index. Since $G / H$ is nilpotent, it is a $p^{\prime}$-group and the center $\zeta(G / H)$ is cyclic (see, for example, [DH, Corollary B.9.4]). Put $\langle g H\rangle=\zeta(G / H)$. Then $M(g-1)=[g, M]$ is a $\mathcal{Z} G$-submodule of $M$, so that $M=[g, M]$. By Lemma 3.5 $A$ has an ascending series

$$
\langle 1\rangle=A_{0} \leq A_{1}=M \leq A_{2} \leq \cdots \leq A_{\alpha} \leq A_{\alpha+1} \leq \cdots \leq A_{\gamma}=A
$$

of $G$-invariant subgroups, every factor $A_{\alpha+1} / A_{\alpha}$ of which is $G$-chief and isomorphic to $M$ as $\mathcal{Z} G$-module for each $\alpha<\gamma$. In particular, $\left[g A_{\alpha}, A_{\alpha+1} / A_{\alpha}\right]=A_{\alpha+1} / A_{\alpha}$ for each $\alpha<\gamma$. It follows that $[g, A]=A$.

Suppose that $C_{A}(g) \neq\langle 1\rangle$. Let $1 \neq a \in C_{A}(g)$ and put $K=\langle a\rangle^{G}$. By (1) $K$ is finite. Let

$$
\langle 1\rangle=K_{0} \leq K_{1} \leq \cdots \leq K_{s}=K
$$

be a series of $G$-invariant subgroups, every factor of which is $G$-chief. Put $U=K_{j+1}, V=K_{j}$. Since $G / A$ is an $F C$-group, $C / A=C_{G / A}(g A)$ has finite index in $G / A$, and (3) yields that $C$ is subnormal in $G$. Let

$$
C=C_{0} \triangleleft C_{1} \triangleleft \cdots \triangleleft C_{m-1} \triangleleft C_{m}=G
$$

be the respective subnormal series. The finiteness of $U / V$ guarantees the existence of a minimal $C_{m-1}$-invariant subgroup $B / V$ of $U / V$. It follows that there are elements $y_{1}, \ldots, y_{t}$ such that $U / V=B / V \times\left(y_{1}^{-1} B y_{1} / V\right) \times$ $\cdots \times\left(y_{t}^{-1} B y_{t} / V\right)$. In turn, the finiteness of $B / V$ implies that it includes a minimal $C_{m-2}$-invariant subgroup $D / V$. Again there are elements $w_{1}, \ldots, w_{r}$ such that $B / V=D / V \times\left(w_{1}^{-1} D w_{1} / V\right) \times \cdots \times\left(w_{r}^{-1} D w_{r} / V\right)$, and hence

$$
\begin{aligned}
& U / V=D / V \times\left(w_{1}^{-1} D w_{1} / V\right) \times \cdots \times\left(w_{r}^{-1} D w_{r} / V\right) \\
& \left.\quad \times\left(y_{1}^{-1} B y_{1} / V\right) \times\left(y_{1}^{-1} w_{1}^{-1} B y_{1} w_{1} / V\right) \times \cdots \times\left(y_{t}^{-1} w_{r}^{-1} D w_{r} y_{t}\right) / V\right) .
\end{aligned}
$$


Repeating the same arguments after finitely many steps we come to a minimal $C$-invariant subgroup $E / V$ and elements $x_{1}, \ldots, x_{q}$ such that

$$
U / V=E / V \times\left(x_{1}^{-1} E x_{1} / V\right) \times \cdots \times\left(x_{q}^{-1} E x_{q} / V\right) .
$$

Since $g A \in \zeta(C / A),[g, E] / V$ is a $C$-invariant subgroup of $E / V$. Then either $[g, E]=V$ or $[g, E]=E$. Suppose that $[g, E]=V$ and consider $\left[g, x_{j}^{-1} E x j\right]$. We have $x_{j} g x_{j}^{-1}=g z$ where $z \in C_{G}(U / V)$, therefore

$$
\begin{aligned}
{\left[g, x_{j}^{-1} E x_{j}\right] } & =x_{j}^{-1}\left[x_{j} g x_{j}^{-1}, E\right] x_{j} \\
& =x_{j}^{-1}[g z, E] x_{j} \\
& =x_{j}^{-1}\left(z^{-1}[g, E] z[z, E]\right) x_{j} \\
& =x_{j}^{-1} V x_{j}=V .
\end{aligned}
$$

Since this is valid for each $j, 1 \leq j \leq q$, we obtain $[g, U]=V$, which is impossible. This contradiction proves that $[g, E]=E$, and it follows that $\left[g, x_{j}^{-1} E x_{j}\right]=x_{j}^{-1} E x_{j}$ for each $j, 1 \leq j \leq q$. Thus $K$ has an ascending series

$$
\langle 1\rangle=L_{0} \leq L_{1} \leq L_{2} \leq \cdots \leq L_{n}=K
$$

of $C$-invariant subgroups, every factor $L_{j+1} / L_{j}$ of which is $C$-chief and $\langle g\rangle$-eccentric. On the other hand, the series $\langle 1\rangle \leq £ \leq\langle a\rangle \leq K$ has a refinement, the all term of which is a $C$-invariant subgroup and every factor of which is $C$-chief. By Schreier Refinement Theorem factors of this series are isomorphic (as $\mathcal{Z} C$-modules) to factors of the series

$$
\langle 1\rangle=L_{0} \leq L_{1} \leq L_{2} \leq \cdots \leq L_{n}=K .
$$

But each factor of this last series is $\langle g\rangle$-eccentric and we obtain a contradiction. This contradiction proves that $C_{A}(g)=\langle 1\rangle$.

Let $x$ be an arbitrary element of $C$. Then $g x=g a_{1}$ for some element $a_{1} \in A$. By the equation $A=[g, A]$ there is an element $a_{2} \in A$ such that $a_{1}=\left[g, a_{2}\right]$. Thus we have $x^{-1} g x=g a_{1}=g\left[g, a_{2}\right]=a_{2}^{-1} g a_{2}$. It follows that $x a_{2}^{-1} \in C_{C}(g)=R$, so that $G=A R$. We have already proved that $A \cap R=A \cap C_{C}(g)=C_{A}(g)=\langle 1\rangle$, that is $G=A \lambda R$. Let $Q$ be another complement to $A$ in $C$. Then $\zeta(Q)$ has an element $e$ such that $g A=e A$. It follows that $e=g a_{3}$ for some element $a_{3} \in A$. The equation $A=[g, A]$ implies the equation $a_{3}=\left[g, a_{4}\right]$ for some element $a_{4} \in A$, so that $e=g a_{3}=g\left[g, a_{4}\right]=a_{4}^{-1} g a_{4}$. Thus $C_{C}(e)=C_{C}\left(a_{4}^{-1} g a_{4}\right)=a_{4}^{-1} C_{C}(g) a_{4}=a_{4}^{-1} R a_{4}$. 
From $e=g a_{3}$ we obtain that $C_{A}(e)=C_{A}(g)=\langle 1\rangle$. Since $e \in$ $\zeta(Q), Q \leq C_{C}(e)$, and from the equation $C=A Q$ we obtain $C_{C}(e)=$ $Q\left(A \cap C_{C}(e)\right)=Q$, that is $Q=a_{4}^{-1} R a_{4}$. Now we can apply Lemma 3 of [RD 3].

Proposition 3.7. Let $G$ be a group, $A$ a normal abelian periodic subgroup of $G$ such that $A \leq F C(G)$ and $G / A$ is an $F C$-group.

(1) If every pronormal subgroup of $G$ is normal, then $G$ is hypercentral.

(2) If $G$ does not include proper contranormal subgroups, then $G$ is hypercentral.

(3) If $G$ does not include proper abnormal subgroups, then $G$ is hypercentral.

Proof: By Proposition 3.4 $G / A$ is hypercentral. Proposition 2.4 implies the decomposition $A=C \oplus E$ where $C$ is the upper $\mathcal{Z} G$-hypercenter of $A$ (that is the intersection of the upper hypercenter of $G$ with $A$ ), $E$ is a maximal $G$-hypereccentric $\mathcal{Z} G$-submodule of $A$. Suppose that $G$ is not hypercentral. It follows that $E \neq\langle 1\rangle$. Let $M$ be a minimal $G$-invariant subgroup of $E$, then $M$ is $G$-eccentric, that is $C_{G}(M) \neq G$. Choose a $G$-invariant subgroup $D$ of $A$ which is maximal with respect to the following properties: $C \leq D$ and $D \cap M=\langle 1\rangle$. Then $A / D$ is a monolithic $\mathcal{Z} G$-module with the $\mathcal{Z} G$-monolith $M D / D$. Furthermore, $C_{G}(M D / D) \neq G$. By Lemma $3.6 G$ includes a subgroup $S / D$ such that $G / D=A / D \lambda S / D$ and every complement to $A / D$ in $G / D$ is conjugate to $S / D$. Let now $g$ be an arbitrary element of $G$. Then $S^{g} / D$ is a complement to $A / D$ in $G / D$. Put $H / D=\left\langle S / D, S^{g} / D\right\rangle$. The equation $G / D=$ $(A / D)(S / D)$ implies that $H / D=B / D \lambda S / D=B / D \lambda S^{g} / D$ where $B / D=A / D \cap H / D$ is an $H$-invariant (and hence $S$-invariant) subgroup of $A / D$. However every $S$-invariant subgroup of $A / D$ is also $G$-invariant. In other words, $B / D$ is a $\mathcal{Z} G$-submodule of $A / D$. As $A / D, B / D$ is monolithic and its monolith is $H$-eccentric. Using Lemma 3.6 we obtain that every complement to $B / D$ is conjugate with $S / D$. In particular, the subgroups $S / D$ and $S^{g} / D$ are conjugate in $\left\langle S / D, S^{g} / D\right\rangle$. This means that the subgroup $S / D$ is pronormal in $G / D$.

Suppose that every pronormal subgroup is normal. Then $S / D$ is normal in $G$. It follows that $G / D=A / D \neq S / D$, in particular the monolith of $A / D$ is central. This contradiction proves that $E=\langle 1\rangle$ and therefore $G$ is hypercentral.

Suppose that $G$ does not include proper contranormal subgroups and let $U / D=N_{G / D}(S / D), V / D=A / D \cap U / D$. If $a D \in V / D$, $x D \in S / D$, then $[a D, x D] \in S / D \cap A / D=\langle 1\rangle$. In other words, 
$N_{A / D}(S / D)=C_{A / D}(S / D)$. If we assume that $V / D \neq\langle 1\rangle$, then $V / D$ includes $M D / D$, in particular, $M D / D \leq C_{G / D}(S / D)$. But this means that $M D / D$ (and hence $M$ ) is central in $G$, which contradicts its selection. This contradiction proves the equation $S / D=N_{G / D}(S / D)$. We have already proved that $S / D$ is pronormal in $G / D$. Since a normalizer of a pronormal subgroup is abnormal (see, for example, [DH, Lemma I.6.21]), the subgroup $S / D$ is also abnormal. Then $S / D$ is contranormal, and we obtain a contradiction. This contradiction proves (2) and obviously (3).

Proof of Theorem B: Let $T$ be the periodic part of $A$. Choose an element $u \in A \backslash T$ and put $U=\langle u\rangle G$. Then $t(U)$ is finite and $U$ is finitely generated. It follows that there is a number $k \in \mathcal{N}$ such that $E=U^{k}$ is torsion-free. In other words, $A$ includes a non-identity finitely generated torsion-free $G$-invariant subgroup. Let $p$ be a prime; then $E^{p}$ is a nonidentity $G$-invariant subgroup. If $A / E^{p}$ is not periodic, then by above it includes a non-identity torsion-free $G$-invariant subgroup.

Let $M / E^{p}$ be a maximal torsion-free $G$-invariant subgroup of $A / E^{p}$. Then $A / M$ is periodic. By Proposition $3.7 G / M$ is hypercentral. Let $r=r_{0}(E)$, then $|E / E p|=p^{r}$. We have $E \cap M=E^{p}$, that is $|E M / M|=$ $p^{r}$. Since $E M / M$ is a normal subgroup of the hypercentral group $G / H$, the hypercenter with the number $r$ includes $E M / M$. It follows that $[E, \underbrace{G, G, \ldots, G]}_{r}]=\left[E,_{r} G\right] \leq M$. On the other hand, $\left[E,_{r} G\right] \leq E$ since $E$

is a normal subgroup, that is $\left[E,_{r} G\right] \leq E \cap M=E^{p}$. Since this is valid for each prime $p,[E, r G] \leq \cap_{p \in \mathcal{P}} E^{p}$ (here $\mathcal{P}$ is the set of all primes). Since $E$ is a free abelian subgroup, $\cap_{p \in \mathcal{P}} E^{p}=\langle 1\rangle$. Hence $\left[E,_{r} G\right]=\langle 1\rangle$, that is the hypercenter with the number $r$ includes $E$. In other words, if $A$ is not periodic, then $A \cap \zeta(G) \neq\langle 1\rangle$. Using Proposition 3.7 with the help of transfinite induction we can obtain that $G$ is hypercentral.

\section{Proofs of main results. The periodic case}

Lemma 4.1. Let $G$ be a group, $H$ a normal periodic subgroup of $G$ such that $H \leq F C(G)$.

(1) If every pronormal subgroup of $G$ is normal, then $H$ is hypercentral.

(2) If $G$ does not include proper contranormal subgroups, then $H$ is hypercentral.

(3) If $G$ does not include proper abnormal subgroups, then $H$ is hypercentral. 
Proof: Let $K$ be a finitely generated subgroup of $H, L=K^{G}$. Since $H \leq F C(G), L$ is finite. Let $S$ be a Sylow $p$-subgroup of $L, p \in \Pi(L)$. For every element $g \in G$ the subgroup $S^{g}$ is also a Sylow $p$-subgroup of $L$, in particular, it is a Sylow $p$-subgroup of $\left\langle S, S^{g}\right\rangle$. Since $\left\langle S, S^{g}\right\rangle \leq L$, it is finite. It follows that $S$ and $S^{g}$ are conjugate. Thus $S$ is a pronormal subgroup. If every pronormal subgroup of $G$ is normal, $S$ also must be normal in $G$. Since it is valid for every prime $p, L$ is nilpotent, so that $H$ is locally nilpotent. Since $H \leq F C(G), H$ is a hypercentral subgroup. Suppose that $G$ does not include proper contranormal subgroups. Since $S$ is pronormal, $N_{G}(S)$ is abnormal (see, for example, [DH, Lemma I.6.21]), and hence contranormal. It follows that $G=N_{G}(S)$, i.e. $S$ is normal in $G$. As above it follows that $H$ is hypercentral.

Lemma 4.2. Let a group $G$ includes an abelian normal p-subgroup $A$ ( $p$ is a prime number) satisfying the following conditions:

(1) $A \leq F C(G)$.

(2) $G / C_{G}(A)$ is a periodic $p^{\prime}$-group.

If $B$ is a finite $G$-invariant subgroup of $A$ such that $A=B \times C$ for some subgroup $C$, then there is a $G$-invariant subgroup $E$ such that $A=B \times E$.

Proof: Let $\mathcal{L}$ be a local family of finite $G$-invariant subgroups of $A$ including $B$. This family exists since $A \leq F C(G)$. If $L \in \mathcal{L}$, then $L=B \times(L \cap C)$. Since $L$ is finite and $C_{G}(L) \geq C_{G}(A), G / C_{G}(L)$ is a finite $p^{\prime}$-group. By Maschke's Theorem (see, for example, $[\mathbf{R D} \mathbf{4}, 4$, 8.1.2]) there exists a $G$-invariant subgroup $D$ such that $L=B \times D$. Let

$\mathcal{C}(L)=\{D \mid D$ is a $G$-invariant subgroup of $L$ such that $L=B \times D\}$.

If $L, M \in \mathcal{L}, L \leq M, Q \in \mathcal{C}(M)$, then $M=B \times Q$ and $L=$ $B \times(L \cap Q)$. Since $L \cap Q$ is $G$-invariant, $L \cap Q \in \mathcal{C}(L)$. Define the mapping $\pi_{M, L}: \mathcal{C}(M) \rightarrow \mathcal{C}(L)$ by the rule: $Q_{\pi_{M, L}}=Q \cap L, Q \in \mathcal{C}(M)$. Clearly, if $L, M, K \in \mathcal{L}$ and $L \leq M \leq K$, then $\pi_{K, M}, \pi_{M, L}=\pi_{k, L}$. By definition $\pi_{L, L}$ is an identity mapping for each $L \in \mathcal{L}$. In other words, $\{\mathcal{C}(L) \mid L \in \mathcal{L}\}$ is a projection set. Then there exists a complete projection set $\mathcal{P}$ (see, for example, [KU, Chapter 55]). This means, that if $R, Q \in \mathcal{P}, R \in \mathcal{C}(L), Q \in \mathcal{C}(M)$ where $L, M \in \mathcal{L}$ and $L \leq M$, then $Q \cap L=R$. Since $\mathcal{L}$ is a local family, $\mathcal{P}$ is a local family too. It follows that $E=\cup \mathcal{P}$ is a $G$-invariant subgroup. Clearly $E \cap B=\langle 1\rangle$. If $a$ is an arbitrary element of $A$, then $a \in L$ for some $L \in \mathcal{L}$. We have now $L=B \times R$ where $R \in P$. It follows that $A=B \times E$. 
Lemma 4.3. Let a periodic group $G$ includes an abelian normal p-subgroup $A$ ( $p$ is a prime) satisfying the following conditions:

(A) $A \leq F C(G)$.

(B) $G / A$ is a hypercentral $p^{\prime}$-group.

Then the following assertions hold:

(1) If every pronormal subgroup of $G$ is normal, then $H$ is hypercentral.

(2) If $G$ does not include proper contranormal subgroups, then $H$ is hypercentral.

(3) If $G$ does not include proper abnormal subgroups, then $H$ is hypercentral.

Proof: Since $A \leq F C(G), A$ has an ascending $G$-chief series

$$
\langle 1\rangle \leq A_{0} \leq A_{1} \leq \cdots \leq A_{\alpha} \leq A_{\alpha+1} \leq \cdots \leq A \gamma=A
$$

with finite factors. Put $M=A_{1}$. Then $M$ is a minimal $G$-invariant subgroup of $A$. In particular, $M$ is an elementary abelian $p$-subgroup, that is $M \leq B=\Omega_{1}(A)$. By Lemma $4.2 B$ includes a $G$-invariant subgroup $E$ such that $B=M \times E$. Let $R$ be a maximal subgroup with the following properties: $E \leq R$ and $R \cap M=\langle 1\rangle$. Put $U / R=\Omega_{1}(A R)$. If we assume that $U / R \neq M R / R$, then using Lemma 4.2 we come to the existence of a non-identity $G$-invariant subgroup $W / R$ such that $U / R=M R / R \times W / R$. However this contradicts the election of $R$. This contradiction proves the equation $U / R=M R / R$, in particular, $U / R$ is finite. It follows that $A / R$ is a Chernikov subgroup (see, for example, [FL, Theorem 25.1]). Let $D / R$ be the divisible part of $A / R$. Since $G / R$ is periodic, $(G / R) / C_{G / R}(D / R)$ is finite (see, for example, [RD 2, Lemma 3.28]). It follows that $G / R$ is hypercentral-by-finite. By generalized Schur-Zassenhaus Theorem (see, for example, [DM, Theorem 2.4.5]) $G / R=A / R \lambda S / R$ where $S / R$ is the Sylow $p^{\prime}$-subgroup of $G / R$. Let $g \in G$ and consider the subgroup $Y / R=\left\langle S / R,(S / R)^{g R}\right\rangle$. Since $Y / R$ is hypercentral-by-finite, Sylow $p^{\prime}$-subgroups of $Y / R$ are conjugate [SE, Lemma 1.1], in particular, $S / R$ and $(S / R)^{g R}$ are conjugate. It follows that $S / R$ is pronormal.

Suppose that every pronormal subgroup is normal, so $S / R$ is normal in $G / R$. Hence $G / R=A / R \times S / R$ and $G / R$ is hypercentral. In particular, $[G, M] \leq R$. On the other hand, $M$ is a normal subgroup of $G$, therefore $[G, M] \leq M$, that is $[G, M] \leq M \cap R=\langle 1\rangle$. This means that $M \leq \zeta(G)$. Using the same arguments by transfinite induction we can prove that $A_{\alpha+1} / A_{\alpha}$ is a $G$-central factor for each ordinal $\alpha<\gamma$. Consequently $G$ is hypercentral. 
Suppose that $G$ does not include proper contranormal subgroups. Then $N_{G / R}(S / R)$ is abnormal (see, for example, [DH, Lemma I.6.21]), and hence contranormal. It follows that $G / R=N_{G / R}(S / R)$, i.e. $S / R$ is normal in $G / R$. As above it follows that $G$ is hypercentral.

Corollary 4.4. Let a periodic group $G$ includes an abelian normal p-subgroup $A$ ( $p$ is a prime), satisfying the following conditions:

(A) $A \leq F C(G)$.

(B) $G / A$ is a hypercentral group.

Then the following assertion hold:

(1) If every pronormal subgroup of $G$ is normal, then $H$ is hypercentral.

(2) If $G$ does not include proper contranormal subgroups, then $H$ is hypercentral.

(3) If $G$ does not include proper abnormal subgroups, then $H$ is hypercentral.

Proof: Since $G / A$ is hypercentral, $G / A=P / A \times S / A$ where $P / A$ a the Sylow $p$-subgroup of $G / A, S / A$ is a Sylow $p^{\prime}$-subgroup of $G / A$. In particular, $P$ is a normal Sylow $p$-subgroup of $G$. By Lemma 4.3 the subgroup $S$ is also hypercentral, so that $S=A \times R$ where $R$ is a (normal) Sylow $p$-subgroup of $G$. In particular, $G=P \times R$. Since $R$ is hypercentral, $G$ is also hypercentral.

Corollary 4.5. Let a periodic group $G$ includes an abelian normal subgroup A satisfying the following conditions:

(A) $A \leq F C(G)$.

(B) $G / A$ is a hypercentral group.

Then the following assertion hold:

(1) If every pronormal subgroup of $G$ is normal, then $H$ is hypercentral.

(2) If $G$ does not include proper contranormal subgroups, then $H$ is hypercentral.

(3) If $G$ does not include proper abnormal subgroups, then $H$ is hypercentral.

Proof: Since $A \leq F C(G), A$ has an ascending $G$-chief series

$$
\langle 1\rangle \leq A_{0} \leq A_{1} \leq \cdots \leq A_{\alpha} \leq A_{\alpha+1} \leq \cdots \leq A_{\gamma}=A
$$


with finite factors. Consider an arbitrary $G$-chief factor $A_{\alpha+1} / A_{\alpha}$ of this series. Let $\Pi\left(A_{\alpha+1} / A_{\alpha}\right)=\{p\}$ and denote by $S$ a Sylow $p^{\prime}$-subgroup of $A$. Then $A_{\alpha+1} / A_{\alpha}$ is $G$-isomorphic with some $G$-chief factor of $A / S$. By Corollary $4.4 G / S$ is hypercentral, and hence $A_{\alpha+1} / A_{\alpha}$ is $G$-central.

Corollary 4.6. Let a periodic group $G$ includes a soluble normal subgroup $A$, satisfying the following conditions:

(A) $A \leq F C(G)$.

(B) $G / A$ is a hypercentral group.

Then the following assertion hold:

(1) If every pronormal subgroup of $G$ is normal, then $H$ is hypercentral.

(2) If $G$ does not include proper contranormal subgroups, then $H$ is hypercentral.

(3) If $G$ does not include proper abnormal subgroups, then $H$ is hypercentral.

Proof: Since $A$ is soluble, it has a finite series of $G$-invariant subgroups

$$
\langle 1\rangle \leq A_{0} \leq A_{1} \leq \cdots \leq A_{k}=A
$$

with abelian factors. Using Corollary 4.5 and ordinary induction we can prove that $G$ is hypercentral.

Corollary 4.7. Let a periodic group $G$ includes a residual finite normal subgroup A, satisfying the following conditions:

(A) $A \leq F C(G)$.

(B) $G / A$ is a hypercentral group.

Then the following assertion hold:

(1) If every pronormal subgroup of $G$ is normal, then $H$ is hypercentral.

(2) If $G$ does not include proper contranormal subgroups, then $H$ is hypercentral.

(3) If $G$ does not include proper abnormal subgroups, then $H$ is hypercentral.

Proof: By Lemma $4.1 A$ is hypercentral. Let $F$ be a finite $G$-invariant subgroup of $A$. Since $A$ is residually finite, $A$ includes a normal subgroup $H$ of finite index such that $H \cap F=\langle 1\rangle$. Put $L=\operatorname{Core}_{G}(H)$. By Remak's Theorem $A / L \leq \Pi_{g \in G} A / H^{g}$, in particular, $A / L$ is soluble. By Corollary $4.6 \mathrm{G} / L$ is hypercentral. Since $F L / L$ is a finite normal subgroup of $G / L$, there is a number $m$ such that $[\underbrace{G, G, \ldots, G}_{m}, F] \leq L$. On 
the other hand, $F$ is a normal subgroup of $G$, so that $[\underbrace{G, G, \ldots, G}_{m}, F] \leq$ $F$. This implies $[\underbrace{G, G, \ldots, G}_{m}, F]=\langle 1\rangle$. In other words, the hypercenter of $G$ with number $m$ includes $F$. Since $F$ is an arbitrary finite $G$-invariant subgroup of $A$, the inclusion $A \leq F C(G)$ implies that the upper hypercenter of $G$ includes $A$. Since $G / A$ is hypercentral, it follows that $G$ is also hypercentral.

Proof of Theorem $C$ : Let $Z=\zeta(H)$, then $Z$ is also $G$-invariant and $H / Z$ is residually finite (see, for example, [RD 2, Lemma 4.31]). By Corollary $4.7 \mathrm{G} / Z$ is hypercentral. Now we can apply Corollary 4.5 which implies that $G$ is hypercentral.

Proof of Corollary C1: Let

$$
\langle 1\rangle=G_{0} \leq G_{1} \leq \cdots \leq G_{m}=G
$$

be the upper $F C$-central series of a group $G$. We will use induction by $m$. If $m=1$, the result follows from Proposition 3.4. Let we have already proved that $G / G_{1}$ is hypercentral. By Theorem $\mathrm{C} G$ is also hypercentral.

Corollary C2. Let $G$ be a periodic locally soluble group with Chernikov Sylow p-subgroups for all prime $p$. The following statements hold.

(1) If every pronormal subgroup of $G$ is normal, then $G$ is hypercentral.

(2) If $G$ does not include proper contranormal subgroups, then $G$ is hypercentral.

(3) If $G$ does not include proper abnormal subgroups, then $G$ is hypercentral.

Proof: By Kargapolov's Theorem (see, for example, [DM, Theorem 2.5.14]) $G$ includes a normal abelian divisible subgroup $D$ such that $G / D$ is a residually finite group with finite Sylow $p$-subgroup for all prime $p$. Hence every finite factor-group of $G$ is nilpotent, $G / D$ is residually nilpotent. But a locally finite residually nilpotent group is locally nilpotent. Thus $G / D$ is a direct product of its finite Sylow $p$-subgroups, in particular, $G / D$ is an $F C$-group. Hence $D$ is an abelian subgroup with Chernikov Sylow $p$-subgroups for all prime $p, D \leq F C(G)$. Now we can apply Corollary C1.

Lemma 4.8. Let $G$ be a Chernikov group, $D$ the divisible part of $G$. If every proper $G$-invariant subgroup of $D$ is finite and $G$ does not include proper contranormal subgroups, then $D \leq \zeta(G)$. 
Proof: Choose a finite subgroup $F$ such that $G=F D$. Put $H=F^{G}$, then $H$ is a proper normal subgroup of $G$. If we suppose that $H$ is infinite, then $H \cap D$ is infinite. Since $H \cap D$ is $G$-invariant, $H \cap D=D$, that is $D \leq H$. But in this case $H=G$. This contradiction proves that $H$ is finite. In particular, $[G, G]$ is finite, and hence $D \leq \zeta(G)$.

Lemma 4.9. Let $G$ be a Chernikov group. Then $G$ is central-by-finite and nilpotent in each of the following cases:

(1) $G$ has no proper contranormal subgroups.

(2) $G$ is a Baer group.

(3) $G$ is a Fitting group.

Proof: Denote by $D$ the divisible part of $G$. Let $G$ satisfies (1). The subgroup $D$ has a series of $G$-invariant subgroups

$$
\langle 1\rangle=D_{0} \leq D_{1} \leq \cdots \leq D_{m}=D
$$

satisfying the following condition: every proper $G$-invariant subgroup of $D_{j+1} / D_{j}, 0 \leq j \leq m-1$, is finite. Corollary $\mathrm{C} 1$ implies that $G$ is a hypercentral group. By Lemma 4.8 every factor of this series is central. The finite factor-group $G / D$ is nilpotent, hence $G$ is nilpotent. But a nilpotent Chernikov group is central-by-finite (see, for example, [RD 2, Lemma 3.13]).

Suppose now that $G$ satisfies (2). Then $G$ is locally nilpotent (see, for example, [LS, Theorem 2.5.1]). We have $G=D K$ where $K$ is a finite subgroup. In particular, $K$ is nilpotent. Since $G$ is a Baer group, $K$ is subnormal. By Lemma 4 of $[\mathbf{H K}] G$ is nilpotent, and $G$ is central-byfinite.

Finally, if $G$ is a Fitting group, then it is a product of a finitely many normal nilpotent subgroups and by Fitting Theorem (see, for example, [RD 2, Theorem 2.18]) $G$ is nilpotent.

Corollary C2. Let $G$ be a periodic locally soluble group with Chernikov Sylow p-subgroups for all prime $p$. The following statements hold.

(1) If every pronormal subgroup of $G$ is normal, then $G$ is hypercentral.

(2) If $G$ does not include proper contranormal subgroups, then $G$ is hypercentral.

(3) If $G$ does not include proper abnormal subgroups, then $G$ is hypercentral. 
Corollary C3. Let $G$ be a periodic locally soluble group with Chernikov Sylow p-subgroups for all prime $p$. The following statements are equivalent.

(1) $G$ does not include proper contranormal subgroups.

(2) $G=X_{p \in \Pi(G)} G_{p}$ where $G_{p}$ is a central-by-finite nilpotent Chernikov p-subgroup, $p \in \Pi(G)$.

(3) $G$ is a Fitting group.

(4) $G$ is a Baer group.

Proof: $(1) \Rightarrow(2)$. By Corollary $4 G$ is hypercentral, so that $G=$ $\mathrm{X}_{p \in \Pi(G)} G_{p}$, where $G_{p}$ is a Sylow $p$-subgroup of $G, p \in \Pi(G)$. The subgroup $G_{p}$ is isomorphic with some factor-group of $G$, therefore $G_{p}$ has no proper contranormal subgroups. By Lemma $4.9 G_{p}$ is central-by-finite.

$(2) \Rightarrow(3)$ and $(2) \Rightarrow(4)$ are obvious.

$(4) \Rightarrow(2)$ and $(3) \Rightarrow(2)$ follow from Lemma 4.9.

(2) $\Rightarrow(1)$. Let $H$ be a proper subgroup of $G$, then $H=\mathrm{X}_{p \in \Pi(H)} H_{p}$, where $H_{p}$ is a Sylow $p$-subgroup $H, p \in \Pi(H)$. Then there is a number $p$ such that, $H_{p} \neq G_{p}$. Since $G_{p}$ is nilpotent, $H_{p} G_{p} \neq G_{p}$. It follows that $H^{G} \neq G$.

\section{A remark on transitivity of abnormality}

In the group $S_{4}$ the subgroup $A_{4}$ is abnormal. The subgroup $B=$ $\langle(12)\rangle$ is maximal and therefore abnormal in $A_{4}$. However, it is almost obvious that $B$ is not abnormal in $S_{4}$. So, as we mentioned already in the Introduction, in general, abnormality is not a transitive relation. Nevertheless, the following result, which could be considered as a useful and general criterion of abnormality, shows that the class of groups with transitive abnormality is significantly broad. For example, as it follows from Lemma 5.1 below all metaabelian groups posses this property.

Lemma 5.1. Let a group $G$ includes a normal subgroup $H$ such that $G / H$ has no proper abnormal subgroups. If for any abnormal subgroup $B$ the intersection $B \cap H$ is normal in $H$, then abnormality is a transitive relation in $G$.

Proof: If $G$ has no proper abnormal subgroups the statement is trivial. Let $B$ be an abnormal subgroup of $G$. Then $H B \geq B$ is an abnormal subgroup in $G$. Since the image of an abnormal subgroup is abnormal, $H B / H$ is abnormal in $G / H$. However, $G / H$ has no proper abnormal subgroups. Therefore $G=H B$. Let $C$ be an abnormal subgroup in $B$. 
By the lemma conditions $H \cap B=D$ is normal in $B$. Since $D$ is normal in $H, D$ is also normal in $G=H B$. An isomorphism $B / D=B /(H \cap$ $B) \cong H B / H$, implies that $B / D$ has no proper abnormal subgroups. As above since $C$ is abnormal in $B, B=D C$. Therefore $G$ includes the normal subgroup $D$ and the abnormal subgroup $B=D C$, such that $C$ is abnormal in $B$. Let $g$ be an arbitrary element of $G$. Since $B=D C$ is abnormal in $G, g \in\left\langle D C^{g}, D C\right\rangle=D\left\langle C^{g}, C\right\rangle$. Then $g=d c$ where $d \in D, c \in\left\langle C^{g}, C\right\rangle$. Since $C$ is abnormal in $B=D C, d \in\left\langle C^{d}, C\right\rangle$. From $c \in\left\langle C^{g}, C\right\rangle$ it follows that $d=g c^{-1} \in\left\langle C^{g c^{-1}}, C\right\rangle \leq\left\langle C^{g}, C\right\rangle$. It means that $g=d c \in\left\langle C^{g}, C\right\rangle$ and $C$ is abnormal in $G$, because in $G$ an abnormality is transitive.

Theorem D and its Corollaries D1, D2, and D3 follow directly from Lemma 5.1. We can easily obtain Corollary D4 admitting that any $S T$-group is metabelian [RD $\mathbf{1}]$.

\section{References}

[BB] M. S. BA AND Z. I. Borevich, Arrangements of intermediate subgroups, (Russian), in: "Rings and linear groups", (Russian), Kuban. Gos. Univ., Krasnodar, 1988, pp. 14-41.

[BR] R. BAER, Nilpotent groups and their generalizations, Trans. Amer. Math. Soc. 47 (1940), 393-434.

[B-BP] A. Ballester-Bolinches and T. Pedraza, On a class of generalized nilpotent groups, J. Algebra 248(1) (2002), 219-229.

[C] R. W. CARTER, Nilpotent self-normalizing subgroups of soluble groups, Math. Z. 75 (1960/1961), 136-139.

[DM] M. R. DIXon, "Sylow theory, formations and Fitting classes in locally finite groups", Series in Algebra 2, World Scientific Publishing Co., Inc., River Edge, NJ, 1994.

[DH] K. Doerk AND T. HAwkes, "Finite soluble groups", de Gruyter Expositions in Mathematics 4, Walter de Gruyter \& Co., Berlin, 1992.

[deFKS] M. De Falco, L. A. Kurdachenko and I. Ya. Subbotin, Groups with only abnormal and subnormal subgroups, Atti Sem. Mat. Fis. Univ. Modena 46(2) (1998), 435-442.

[F] A. Fattahi, Groups with only normal and abnormal subgroups, J. Algebra 28 (1974), 15-19. 
[FL] L. Fuchs, "Infinite abelian groups", Vol. I, Pure and Applied Mathematics 36, Academic Press, New York-London, 1970.

[HK] H. Heineken and L. A. Kurdachenko, Groups with subnormality for all subgroups that are not finitely generated, Ann. Mat. Pura Appl. (4) 169 (1995), 203-232.

[KW] O. H. Kegel AND B. A. F. Wehrfritz, "Locally finite groups", North-Holland Mathematical Library 3, NorthHolland Publishing Co., Amsterdam-London; American Elsevier Publishing Co., Inc., New York, 1973.

[KUS] L. A. Kurdachenko and I. Ya. Subbotin, On transitivity of pronormality, Comment. Math. Univ. Carolinae 43(4) (2002), 583-594.

[KU] A. G. Kurosh, "The theory of groups", Translated from the Russian and edited by K. A. Hirsch, 2nd English ed., 2 volumes, Chelsea Publishing Co., New York, 1960.

[KS 1] N. F. Kuzenny I And I. YA. Subbotin, Groups in which all of the subgroups are pronormal, (Russian), Ukrain. Mat. Zh. 39(3) (1987), 325-329, 405; translation in: Ukrainian Math. J. 39 (1987), 251-254.

[KS 2] N. F. Kuzenny ̌ And I. Ya. Subbotin, Groups with pronormal primary subgroups, (Russian), Ukrain. Mat. Zh. 41(3) (1989), 323-327, 428; translation in: Ukrainian Math. J. 41(3) (1989), 286-289.

[KS 3] N. F. Kuzenny ̌ And I. YA. Subbotin, New characterizations of locally nilpotent $\overline{\mathrm{IH}}$-groups, (Russian), Ukrain. Mat. Zh. 40(3) (1988), 322-326, 406; translation in: Ukrainian Math. J. 40(3) (1988), 274-277 (1989).

[LS] J. C. Lennox And S. E. Stonehewer, "Subnormal subgroups of groups", Oxford Mathematical Monographs, Oxford Science Publications, The Clarendon Press, Oxford University Press, New York, 1987.

[McL] D. H. MCLAIN, Remarks on the upper central series of a group, Proc. Glasgow Math. Assoc. 3 (1956), 38-44.

[MW] W. MöHrEs, Auflösbarkeit von Gruppen, deren Untergruppen alle subnormal sind, Arch. Math. (Basel) 54(3) (1990), $232-235$.

[M] K. H. MüLlER, Schwachnormale Untergruppen: Eine gemeinsame Verallgemeinerung der normalen und normalisatorgleichen Untergruppen, Rend. Sem. Mat. Univ. Padova 36 (1966), $129-157$. 
[P] T. A. Peng, Pronormality in finite groups, J. London Math. Soc. (2) 3 (1971), 301-306.

[PB] B. I. Plotkin, On the theory of locally nilpotent groups, (Russian), Doklady Akad. Nauk SSSR (N.S.) 76 (1951), 639-641.

[RD 1] D. J. S. Robinson, Groups in which normality is a transitive relation, Proc. Cambridge Philos. Soc. 60 (1964), 21-38.

[RD 2] D. J. S. RoBinson, "Finiteness conditions and generalized soluble groups", Part 1, Ergebnisse der Mathematik und ihrer Grenzgebiete 62, Springer-Verlag, New York-Berlin, 1972; Part 2, Ergebnisse der Mathematik und ihrer Grenzgebiete 63, Springer-Verlag, New York-Berlin, 1972.

[RD 3] D. J. S. Robinson, Splitting theorems for infinite groups, in: "Symposia Mathematica", Vol. XVII (Convegno sui Gruppi Infiniti, INDAM, Rome, 1973), Academic Press, London, 1976, pp. $441-470$.

[RD 4] D. J. S. Robinson, "A course in the theory of groups", Graduate Texts in Mathematics 80, Springer-Verlag, New YorkBerlin, 1982.

[R 1] J. S. Rose, Nilpotent subgroups of finite soluble groups, Math. Z. 106 (1968), 97-112.

[R 2] J. S. Rose, Finite soluble groups with pronormal system normalizers, Proc. London Math. Soc. (3) 17 (1967), 447-469.

[SH 1] H. Smith, Hypercentral groups with all subgroups subnormal, Bull. London Math. Soc. 15(3) (1983), 229-234.

[SH 2] H. Smith, A note on locally soluble, normal closures of cyclic subgroups, Glasgow Math. J. 36(1) (1994), 33-36.

[SH 3] H. Sмiтh, Torsion-free groups with all subgroups subnormal, Arch. Math. (Basel) 76(1) (2001), 1-6.

[SE] S. E. STONEHEWER, Abnormal subgroups of a class of periodic locally soluble groups, Proc. London Math. Soc. (3) 14 (1964), $520-536$.

[SK] I. Ya. Subbotin And N. F. KuzennyĬ, Locally solvable groups in which all infinite subgroups are pronormal, (Russian), Izv. Vyssh. Uchebn. Zaved. Mat. no. 11 (1988), 77-79; translation in: Soviet Math. (Iz. VUZ) 32(11) (1988), 126-131.

[W] G. J. Wood, On pronormal subgroups of finite soluble groups, Arch. Math. (Basel) 25 (1974), 578-585. 
Leonid A. Kurdachenko:

Mathematics Department

Dnepropetrovsk University

Vulytsya Naukova 13

49050 Dnepropetrovsk

Ukraine

E-mail address: mmf@ff.dsu.dp.ua

E-mail address: lkurdachenko@ua.fm

Igor Ya. Subbotin:

Mathematics Department

National University

9920 S. La Cienega Blvd

Inglewood, CA 90301

U.S.A.

E-mail address: isubboti@nu.edu

Primera versió rebuda el 10 de setembre de 2002, darrera versió rebuda el 28 d'abril de 2003. 